\title{
What is the value of musculoskeletal ultrasound in patients presenting with arthralgia to predict inflammatory arthritis development? A systematic literature review
}

Rosaline van den Berg ${ }^{1 *}$ D, Sarah Ohrndorf ${ }^{2,3}$, Marion C. Kortekaas ${ }^{2}$ and Annette H. M. van der Helm-van Mil ${ }^{1,2}$

\begin{abstract}
Objective: Musculoskeletal ultrasound (US) is frequently used in several rheumatology practices to detect subclinical inflammation in patients with joint symptoms suspected for progression to inflammatory arthritis. Evaluating the scientific basis for this specific US use, we performed this systematic literature review determining if US features of inflammation are predictive for arthritis development and which US features are of additive value to other, regularly used biomarkers.
\end{abstract}

Methods: Medical literature databases were systematically searched up to May 2017 for longitudinal studies reporting on the association between greyscale (GSUS) and Power Doppler (PDUS) abnormalities and inflammatory arthritis development in arthralgia patients. Quality of studies was assessed by two independent reviewers using a set of 18 criteria. Studies were marked high quality if scored $\geq 80.6 \%$ (which is the median score). Best-evidence synthesis was performed to determine the level of evidence (LoE). Positive and negative likelihood ratios ( $L R+, L R-$ ) were determined.

Results: Of 3061 unique references, six fulfilled inclusion criteria (three rated high quality), of which two reported on the same cohort. Heterogeneity in arthralgia populations, various US machines and scoring systems hampered the comparability of results. LoE for GSUS as predictor was limited and moderate for PDUS; LoE for the additive value of GSUS and PDUS with other biomarkers was limited to moderate. Estimated LR+ values were mostly $<4$ and $L R-$ values $>0.5$.

Conclusions: Data on the value of GSUS and PDUS abnormalities for predicting inflammatory arthritis development are sparse. Although a potential benefit is not excluded, current LoE is limited to moderate. Future studies are required, preferably performed in clearly defined, well-described arthralgia populations, using standardized US acquisition protocols and scoring systems.

Keywords: Arthralgia, Ultrasound, Rheumatoid arthritis

\footnotetext{
* Correspondence: rosalinevandenberg@gmail.com

'Department of Rheumatology, Erasmus Medical Center, Rotterdam, The

Netherlands

Full list of author information is available at the end of the article
}

(c) The Author(s). 2018 Open Access This article is distributed under the terms of the Creative Commons Attribution 4.0 International License (http://creativecommons.org/licenses/by/4.0/), which permits unrestricted use, distribution, and reproduction in any medium, provided you give appropriate credit to the original author(s) and the source, provide a link to the Creative Commons license, and indicate if changes were made. The Creative Commons Public Domain Dedication waiver (http://creativecommons.org/publicdomain/zero/1.0/) applies to the data made available in this article, unless otherwise stated. 


\section{Background}

The development of rheumatoid arthritis (RA) is supposed to consist of several stages: a) genetic risk factors for RA; b) environmental risk factors for RA; c) systemic autoimmunity associated with RA; d) symptoms without clinical arthritis; e) unclassified arthritis (UA); f) RA [1]. The phase of arthralgia preceding clinical arthritis (phase d) is of particular interest since it is hypothesized that disease-modifying treatment initiated in this phase might result in better disease outcomes than when initiated in the phases of UA and RA [2]. However, musculoskeletal symptoms such as arthralgia are prevalent, and arthralgia is frequently not related to imminent RA. In order to identify arthralgia patients at risk for RA, different strategies can be undertaken, such as selecting arthralgia patients based on clinical features associated with RA development, using autoantibody tests or imaging to detect subclinical inflammation, or a combination of these.

Musculoskeletal ultrasound (US) is a frequently used imaging modality as it is fast, easy to apply, and readily accessible. Although US is frequently used in patients presenting with arthralgia (as also proposed in an algorithm for the pragmatic use of US [3]) in several rheumatology practices, we questioned what the scientific basis is to use US as a predictor for future inflammatory arthritis development. Therefore, we systematically studied the literature to determine if US features of inflammation are predictive for inflammatory arthritis development and, if so, to determine which US features are of additive value to other regularly used biomarkers, with the ultimate goal of obtaining evidence-based information on the value of US in patients presenting with arthralgia.

\section{Methods}

\section{Systematic literature search}

The PRISMA guidelines were followed [4]. Search strategies were built in collaboration with an experienced librarian (WB) and executed in electronic medical literature databases (Embase.com, Medline Ovid, Web of Science, Scopus, Cochrane Central, Google Scholar) up to 11 May 2017 (complete searches in Additional file 1: File S1). Reference lists of the included papers were checked for additional papers and unpublished and ongoing trials were identified using the World Health Organization (WHO) International Clinical Trials Registry Platform (ICTRP) search portal (http://apps.who.int/trialsearch/) and ClinicalTrials.gov (http://clinicaltrials.gov).

\section{Selection of studies based on inclusion and exclusion criteria}

Two reviewers (SO, RvdB) assessed each title for suitability for inclusion in this review, according to predetermined inclusion and exclusion criteria. Next, abstracts were retrieved for detailed review and, finally, full-text papers were assessed if further information was required. Papers not addressing the topic of interest were excluded and reasons for exclusion recorded.

From the total number of studies identified by the database search, studies were included if the following inclusion criteria were met: 1) investigation of subjects without clinical arthritis, suffering from arthralgia, regardless of rheumatoid factor (RF) and anti-citrullinated protein antibody (ACPA) status or ACPA+ musculoskeletal symptoms; 2) investigation of small hand and/or feet joints of subjects using US; 3) joints and/or tendons were assessed for inflammatory features (GS synovial hypertrophy and/or PDUS); 4) subjects were followed prospectively; 5) development of (persistent) inflammatory arthritis or RA was defined as outcome. Studies about other inflammatory joint conditions, animal studies, reviews, letters to the editor, case reports, case series, commentaries, guidelines, editorials, abstracts, study populations < 18 years of age, and studies in languages other than English, Dutch, and German were excluded.

\section{Data extraction}

The two reviewers independently assessed the full texts of the included studies using a predefined sheet to extract data about: 1) study population (number of patients, age, gender, symptom duration); 2) follow-up period; 3) musculoskeletal US equipment (producer, transducer, machine setting, mode (GSUS/PDUS); 4) US acquisition (number and type of examined joints, examined pathology, scoring method, potential used cut-off); 5) longitudinal outcome.

Data from univariable analyses were extracted to answer the first aim; data from multivariable analyses were extracted to answer the second aim on added value.

\section{Quality assessment and analyses}

Due to heterogeneity of the studies, it was not possible to perform meta-analyses and calculate pooled effect estimates. Therefore, we performed a best-evidence synthesis based on the guidelines on systemic review of the Cochrane Collaboration Back and Neck (CBN) Group [5], a method summarizing the level of evidence (LoE) in observational studies if study population, outcomes and data analyses are heterogenic (Additional file 1: Table S1). LoE is based on presence of statistical significance, which depends on sample sizes, taking into account the quality of the studies. Quality of the studies was evaluated by the two reviewers individually, using a set of 18 criteria based on previous systematic reviews in prognostic factors in the field of musculoskeletal disorders $[2,6]$. This list included seven criteria specifically for the use of US, of which three were considered mandatory (Additional file 1: Table S2). A study was considered high quality if all three mandatory criteria 
were fulfilled and the total score was $\geq 80.6 \%$ (median of quality scores obtained in this review).

Positive and negative likelihood ratios (LR+ and LR-, respectively) and positive and negative predictive values (PPV and NPV, respectively) were calculated based on presented data regarding outcome (using the presented follow-up duration (Table 1)) to evaluate the predictive accuracy. Also, due to heterogeneity, no summary estimates were calculated.

\section{Results}

\section{Selection and inclusion of articles}

In total, 5028 titles were identified and, after removing duplicates, 3061 unique references were screened (Additional file 1: Figure S1). After detailed review, six full-text papers fulfilled the inclusion and exclusion criteria (Table 1) [7-12], of which two studies reported on the same cohort $[10,11]$. One of them reports on dichotomous PDUS results only and the other presents PDUS and GS synovial hypertrophy results for various cut-offs.

\section{Quality assessment}

The two reviewers rated 108 items and agreed on 98 (91.6\%); disagreement on items was solved by discussion (Additional file 1: Table S3). All six included studies fulfilled the three mandatory criteria. Median quality score was $80.6 \%$ (range 61.1-83.3\%). Two of the three high-quality papers described the same cohort $[8,10,11]$.

\section{Study characteristics}

The number of included patients varied between 80 and 379 ; the majority were female $(69-83 \%)$ aged $>50$ years. None of the studies had stringent inclusion criteria with respect to symptom constitution. The cohort described in the papers by Nam et al. [10] and Rakieh et al. [11] included ACPA+ patients with new onset musculoskeletal symptoms from primary care physician clinics and the rheumatology early arthritis clinic in Leeds. In the study of Van der Ven et al. [8], patients with inflammatory joint complaints involving at least two joints in the hands, feet, or shoulders for $<1$ year which could not be explained by other conditions were included if they had also at least two of the following criteria: morning stiffness for $>1 \mathrm{~h}$, unable to clench a fist in the morning, pain when shaking someone's hand, pins and needles in the fingers, difficulties wearing rings or shoes, family history of RA, and/or unexplained fatigue. In the paper by Zufferey et al. [7], ACPA- and RF-negative patients with polyarthralgia for $>6$ weeks with an inflammatory or mixed (mechanical and inflammatory) character referred by their general practitioner or rheumatologist were included. Van de Stadt et al. [12] recruited ACPA+ and/or $\mathrm{RF}+$ patients with arthralgia, defined as "non-traumatic pain in any joint", at rheumatology clinics in Amsterdam after referral by their general practitioner. Patients presenting with new-onset arthralgia to the Newcastle Early Arthritis Clinic were included in the study by Pratt et al. [9], but no description of arthralgia was provided.

Symptom duration at inclusion varied between 6 weeks and 23 months (Table 1). Patients were followed for > 12 months in all studies (range 12-28 months). Three studies included only ACPA+ and/or RF+ patients [1012]; one study only ACPA- and RF-negative patients [7] and the remaining studies included both ACPA+ and/or $\mathrm{RF}+$ and arthralgia negative patients $[8,9]$.

\section{Acquisition of ultrasound}

US specifications are presented in Table 2. Three studies used a transducer with 12 or $13 \mathrm{MHz}$ as maximum [7, 9, 12]. Various US machines were used, various scoring systems with various definitions of pathology were used to grade synovitis [13-20], and the number of examined joints varied (range 16-32). In one study only tender joints were scanned [12]. Four studies reported on both GS synovial hypertrophy and PDUS [8-10, 12], one only on GS synovial hypertrophy [7], and one only on PDUS [11]. Only one study scored the presence of tenosynovitis (GSUS) [12]. All studies except one [10] used a cut-off to define a positive "inflammation US score", yet the definitions varied (Table 2).

Two studies reported on inter-observer reliability, which was moderate (kappa $=0.56$ for GS synovial hypertrophy) to substantial (kappa $=0.64$ for PDUS) [9] in one study, and fair (kappa $=0.22$ for effusion) to moderate (kappa $=0.47$ for synovitis) and substantial (kappa $=0.67$ for PDUS) in another study [12], yet good in terms of overall percentage agreement (88-92\%).

\section{Outcome}

Outcome was defined as RA (ACR/EULAR 2010 criteria [21]) in one study and (persistent) (inflammatory) arthritis in the remaining five. Outcome was reached in 8.8$50.0 \%$ of patients; frequency was lowest in ACPA-/ RF-negative populations and highest in $\mathrm{ACPA}+/ \mathrm{RF}+$ populations. Duration until outcome was reached varied between 7.9 and 18.3 months and was not specified in two studies (Table 1).

\section{LoE of GSUS and PDUS abnormalities as predictor for arthritis development}

The prevalence of different US features varied per patient group and cut-off used. For GS synovial hypertrophy it ranged from 11.6 (GSUS $\geq 2$ in patients without arthritis development) to $77.2 \%$ (GSUS $\geq 2$ in patients that developed arthritis); for PDUS from 6.3 (PDUS $=2$ in patients without arthritis development) to $44.0 \%$ (PDUS $\geq 1$ in patients that developed arthritis) 


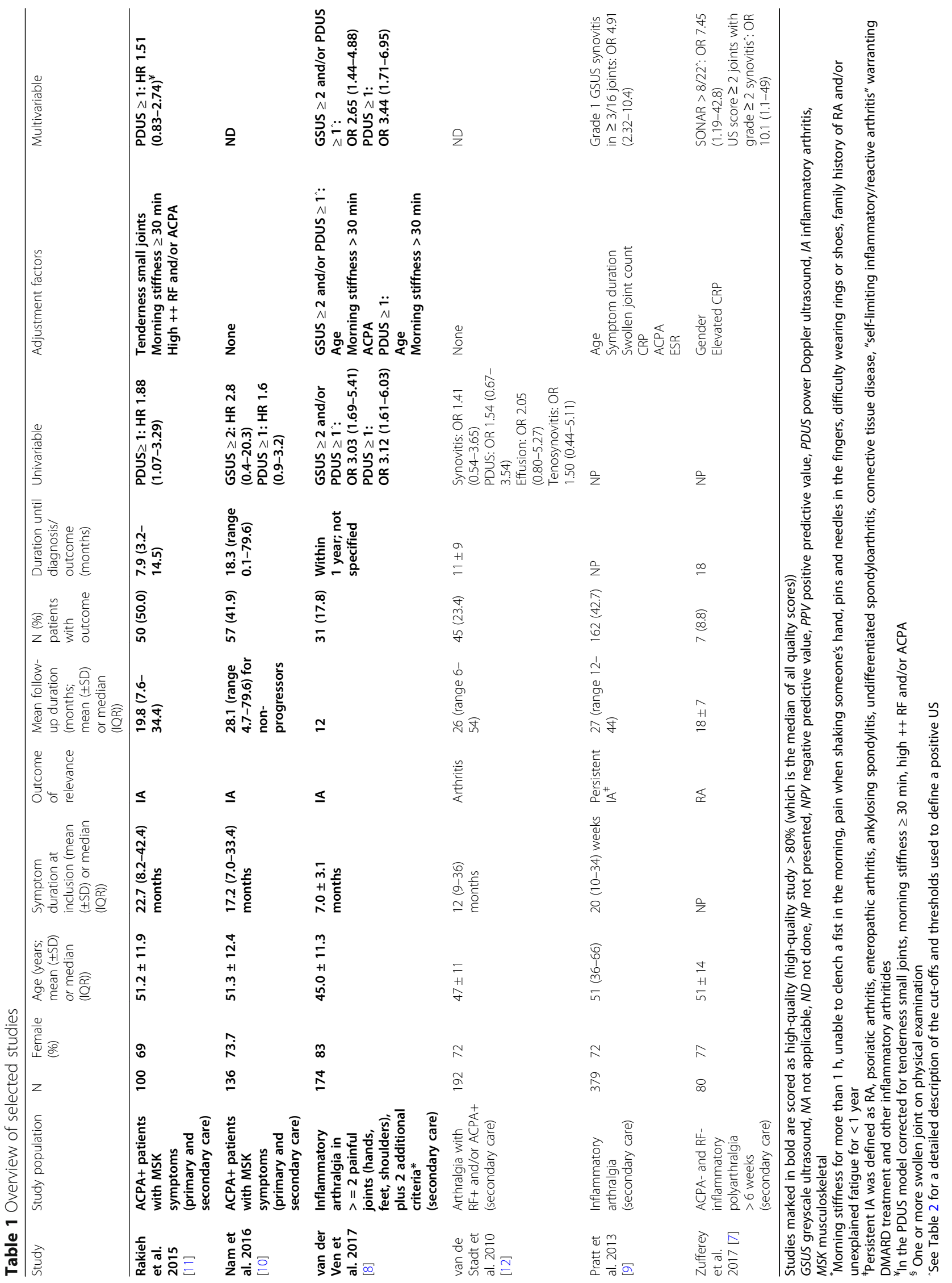




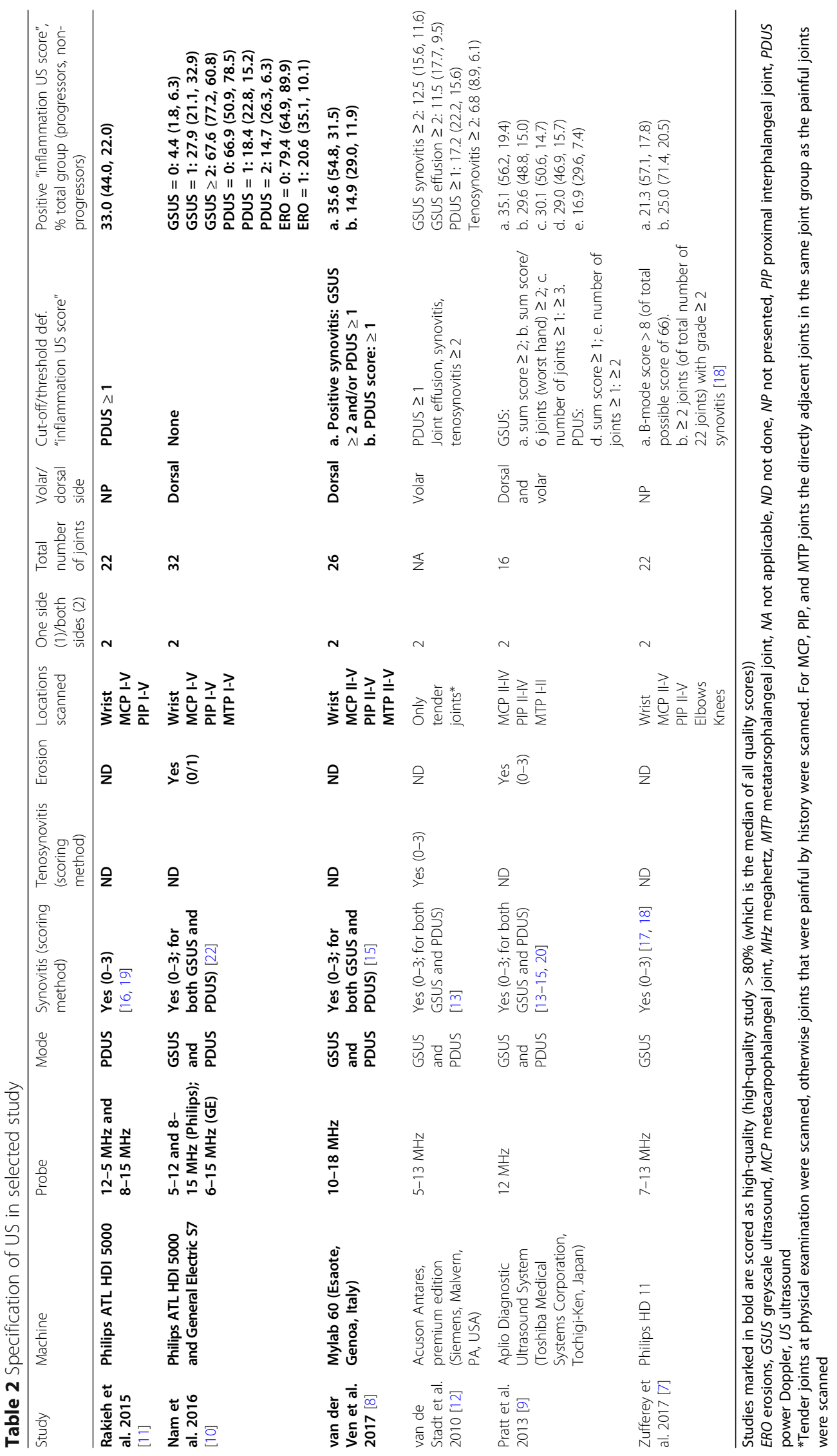


(Table 2). The prevalence of tenosynovitis ranged from 6.1 (GSUS $\geq 2$ in patients without arthritis development) to $8.9 \%$ (GSUS $\geq 2$ in patients with arthritis development).

\section{GS synovial hypertrophy}

One high-quality and one low-quality study reported a non-statistically significant association between GS synovial hypertrophy and arthritis development (HR 2.8 [95\% CI 0.4-20.3] and (OR 1.41 [95\% CI 0.54-3.65], respectively) $[10,12]$. One other high-quality study reported a statistically significant association (OR 3.03 [95\% CI: 1.69-5.41]) for a "positive US" defined as GSUS $\geq 2$ and/or PDUS $\geq 1$ [8]. Hence, LoE with regard to the predictive value of GSUS is limited.

\section{PDUS synovitis}

Two high-quality studies reported a statistically significant association between PDUS and arthritis development (OR 3.12 [95\% CI 1.61-6.03] [8], HR 1.88 [95\% CI 1.07-3.29] [11]). The third high-quality study (performed in the same cohort as [11]) reported a non-statistically significant association (HR 1.6 [95\% CI 0.9-3.2]) [10]; thus the statistically significant association found in the first 100 patients was lost after inclusion of additional patients. A low-quality study reported a non-significant association as well (OR 1.54 [95\% CI 0.67-3.54]) [12]. Hence, LoE with regard to the predictive value of PDUS is moderate.

\section{Tenosynovitis}

One low-quality study evaluated tenosynovitis and found no statistically significant association with arthritis development (OR 1.50 [95\% CI 0.44-5.11]) [12]. Hence, LoE with regard to the predictive value of tenosynovitis is insufficient.

\section{LoE of GSUS and PDUS abnormalities being additive to other biomarkers}

Three studies investigated the association of GS synovial hypertrophy with arthritis development, correcting for different biomarkers (Table 1). Two low-quality studies reported statistically significant associations of GS synovial hypertrophy and arthritis development (OR 4.91 [95\% CI 2.32-10.4]), OR 7.45 [95\% CI 1.19-42.8], and OR 10.1 [95\% CI 1.1-49] [7, 9]. One high-quality study reported a statistically significant association of a "positive US" (GSUS $\geq 2$ and/or PDUS $\geq 1$; OR 2.65 [95\% CI 1.44-4.88]) [8]. Hence, LoE with regard to the question of whether GS synovial hypertrophy may have value in predicting arthritis development, additive to regularly assessed biomarkers, is moderate.

Likewise, two studies performed multivariable analysis with PDUS. After correction for (different) biomarkers
(Table 1), one high-quality study reported a statistically significant association (OR 3.44 [95\% CI 1.71-6.95]) [8]. The other high-quality study reported a non-significant association (HR 1.51 [95\% CI 0.83-2.74]) [11]. Hence, LoE of the value of PDUS in addition to other biomarkers is limited.

The value of tenosynovitis (GS/PD) in addition to other biomarkers was not investigated.

Positive and negative likelihood ratios and absolute risks Calculated LRs varied and confidence intervals (CIs) were wide. For GS synovial hypertrophy, LR+ ranged from 1.27-3.48 and LR- ranged from 0.36-0.95. For PDUS, LR+ ranged from 1.42-4.16 and LR- ranged from 0.63-0.92 (Fig. 1 and Additional file 1: Table S4).

Predictive values are directly proportional to disease prevalence. Percentages of patients that developed arthritis varied between 8.8 and 50\%; thus, prior risks for not progressing were $50-91.2 \%$. We calculated the increase in the absolute risks of inflammatory arthritis provided by US-detected abnormalities by comparing PPV and NPV with prior risks (Additional file 1: Table S4). Overall, PPVs were low or moderate (23.5-71.9\% for GS synovial hypertrophy; $30.3-75 \%$ for PDUS) and the increase in absolute risks in US-positive patients ranged from 5.8-29.2\% (GS synovial hypertrophy) and 6.9-33.1\% (PDUS). NPVs were higher (68.9-96.7\% for GS synovial hypertrophy; 58.2$85.1 \%$ for PDUS), but the gain in relation to prior risk of not progressing to arthritis was relatively small (0.8-12.5\% for GS synovial hypertrophy; 2.9-13.9\% for PDUS). Thus, NPVs were largely explained by prior risks of not developing inflammatory arthritis.

\section{Discussion}

The aim of this systematic literature review was to determine if US features of inflammation are predictive for inflammatory arthritis development and, if so, which US features are of additive value to other regularly used biomarkers. LoE for GS synovial hypertrophy as predictor for arthritis was limited and moderate for PDUS. LoE for the additive value of GS synovial hypertrophy and PDUS with other regularly used biomarkers was limited to moderate. Additionally, there was insufficient data on the value of US-detected tenosynovitis. Thus, there is a discrepancy between the frequent use of US in arthralgia patients to search for subclinical inflammation (which, if present, is generally considered a sign of imminent RA) in several rheumatology practices and the absence of strong scientific evidence on its prognostic value.

The limited/moderate LoE might be explained by relatively low number of studies and the presence of different types of heterogeneity. Only six studies were included in this systematic literature review, of which two described the same cohort. The number of included 


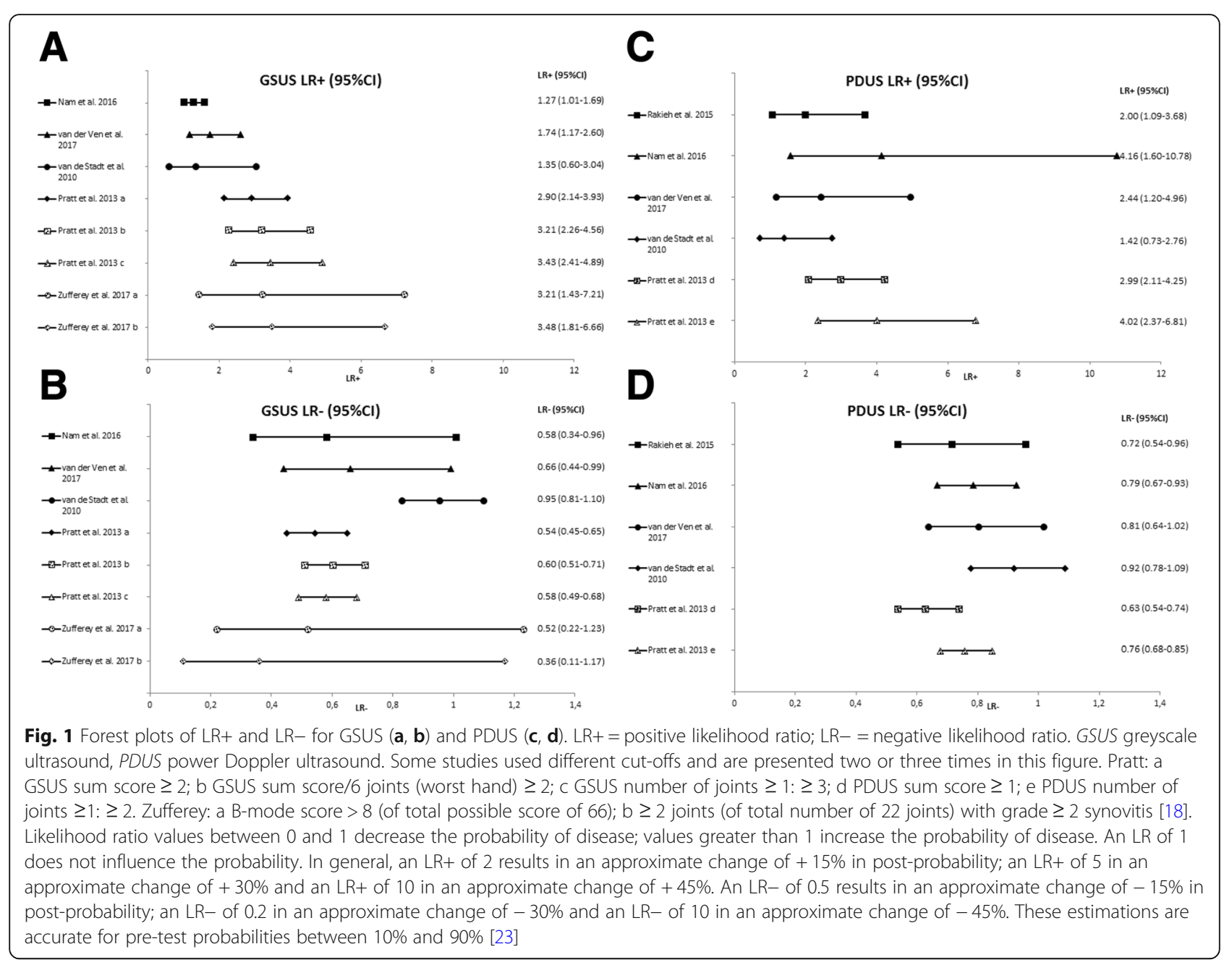

patients per study was rather low, influencing the power to achieve statistical significance. Furthermore, heterogeneous arthralgia populations (seropositive arthralgia, seronegative arthralgia, ACPA+ patients with unspecific musculoskeletal (MSK) symptoms) were studied in different settings (primary and/or secondary care), with slightly differently defined outcomes ((persistent) (inflammatory) arthritis, RA), contributing to the various ranges of frequencies of outcome (8.8-50\%).

Moreover, the US acquisition protocol, definitions of pathology, and scoring systems varied, although all followed internationally recognized recommendations and scoring systems [13-20]. Only very recently, EULAR/ OMERACT published a standardized, consensus-based semi-quantitative scoring system for GS synovial hypertrophy and PDUS (separately and combined) [24, 25], but this was not available when the studies included in this review were executed.

Other sources of heterogeneity were the selection of assessed joints, whether they were scanned from a volar or dorsal aspect, and the fact that different machines were used. It is known that the diverse machines have a wide variation in sensitivity to pick up inflammation, especially with regard to Doppler modalities [26]. Three studies used a transducer with 12 or $13 \mathrm{MHz}$ as maximum, while higher frequencies are recommended especially for scanning small hand joints. Ideally, in order to arrive at a higher LoE, future studies should be performed in more homogeneous arthralgia populations (e.g., fulfilling the EULAR definition of arthralgia at risk for RA [27]), using the same scan and scorings protocols (e.g., EULAR/ OMERACT [24, 25]).

Another issue is the definition of a "positive US". Different cut-offs were applied and none of the studies included information on US findings in healthy volunteers. It has been shown that a cut-off incorporating such findings increased the prognostic value for the use of MRI in arthralgia patients [28]. Also US "inflammatory features" can be detected in healthy volunteers, especially in certain joints and increasing with age [29-36]. Whether incorporating age-dependent US reference values might increase the predictive value of US remains to be determined. 
There was insufficient data to determine whether US-detected tenosynovitis is an (important) predictor of arthritis development, which is the case for MRI-detected subclinical tenosynovitis (which is an even stronger predictor than MRI-detected subclinical synovitis or bone marrow edema) [37]. Therefore, the potential of US-detected tenosynovitis requires further investigation.

We sought to explore the value of US abnormalities in addition to other frequently used predictors of arthritis development. Some studies performed multivariable analyses but adjusted for different variables; hence, the results of these multivariable analyses could not be directly compared. Further studies on this subject are needed, also using methods such as net reclassification index.

Best-level evidence synthesis focuses on statistical significance. Since this is not directly applicable for clinical practice, we also expressed prognostic accuracy using LRs. Estimated LR+ values were mostly $<4$ and LRvalues $>0.5$, some with wide CIs, indicating that the post-test probability was altered to only a small degree. This was also observed when we calculated increases in absolute risks (comparing pre-test with observed post-test risks). Although absolute NPVs were higher than PPVs, and seemingly more informative, this was caused by the prior risks, which were relatively low. Our comparison of pre-test and post-test risks suggested that US is slightly more helpful in "ruling in" than "ruling out" imminent inflammatory arthritis.

\section{Conclusions}

US is frequently used in arthralgia patients in several rheumatologic practices, and although some studies have suggested a potential benefit of US, the current LoE is limited to moderate at best, due to heterogeneity of studies and lack of replication. Yet, there is a strong need for validation of results in future US studies, preferably performed in clearly defined, well-described arthralgia patients. The EULAR definition of arthralgia suspicious for progression to RA might be used to this end.

\section{Additional file}

Additional file 1: Overview of literature research, Best-evidence synthesis, Criteria and scores of the quality assessment, Calculated PPVs \& NPVs and increase in absolute risks, Search strategies for each database. (DOCX 85 kb)

\section{Abbreviations}

ACPA: Anti-citrullinated protein antibody; ACR: American College of Rheumatology; Cl: Confidence interval; EULAR: European League Against Rheumatology; GS: Greyscale; GSUS: Greyscale ultrasound; HR: Hazard ratio; ICTRP: International Clinical Trials Registry Platform; LoE: Level of evidence; LR - : Negative likelihood ratio; LR+: Positive likelihood ratio; MHz: Megahertz; MRI: Magnetic resonance imaging; NPV: Negative predictive value; OMERACT: Outcome measures in rheumatology; OR: Odds ratio; PD: Power Doppler; PDUS: Power Doppler ultrasound; PPV: Positive predictive value;
RA: Rheumatoid arthritis; RF: Rheumatoid factor; UA: Unclassified arthritis; US: Ultrasound; WHO: World Health Organization

\section{Acknowledgements}

We would like to thank Wichor Bramer from the Medical Library of the Erasmus Medical Center for helping us to build the search strategies.

\section{Funding}

This work was supported by the Dutch Arthritis Foundation.

The work of Sarah Ohrndorf was supported by the Articulum fellowship grant from Pfizer (Vienna, Austria) and by the BMBF (German ministry for education and research) funded project 'ArthroMark'.

\section{Availability of data and materials}

Data sharing is not applicable as no datasets were generated or analyzed during the current study.

\section{Authors' contributions}

RvdB, SO, MCK, and AHMvdH-vM contributed to the conception and design of the review. RvdB performed the literature search. RvdB and SO assessed all papers and performed the data extraction and quality assessment. RvdB performed the analyses. RvdB and AHMvdH-vM drafted the paper. MCK and SO revised the article for important intellectual content. All authors gave final approval of the version to be published.

\section{Ethics approval and consent to participate}

NA

\section{Consent for publication}

NA

Competing interests

The authors declare that they have no competing interests.

\section{Publisher's Note}

Springer Nature remains neutral with regard to jurisdictional claims in published maps and institutional affiliations.

\section{Author details}

1Department of Rheumatology, Erasmus Medical Center, Rotterdam, The Netherlands. ${ }^{2}$ Department of Rheumatology, Leiden University Medical Center, Leiden, The Netherlands. ${ }^{3}$ Department of Rheumatology and Clinical Immunology, Charité - Universitätsmedizin Berlin, Berlin, Germany.

Published online: 11 October 2018

\section{References}

1. Gerlag DM, Raza K, van Baarsen LG, et al. EULAR recommendations for terminology and research in individuals at risk of rheumatoid arthritis: report from the Study Group for Risk Factors for Rheumatoid Arthritis. Ann Rheum Dis. 2012;71:638-41.

2. van Nies JA, Krabben A, Schoones JW, et al. What is the evidence for the presence of a therapeutic window of opportunity in rheumatoid arthritis? A systematic literature review. Ann Rheum Dis. 2014:73:861-70.

3. D'Agostino MA, Terslev L, Wakefield $R$, et al. Novel algorithms for the pragmatic use of ultrasound in the management of patients with rheumatoid arthritis: from diagnosis to remission. Ann Rheum Dis. 2016;75:1902-8.

4. Moher D, Liberati A, Tetzlaff J, et al. Preferred reporting items for systematic reviews and meta-analyses: the PRISMA statement. J Clin Epidemiol. 2009;62:1006-12.

5. Furlan AD, Malmivaara A, Chou R, et al. 2015 Updated method guideline for systematic reviews in the Cochrane Back and Neck Group. Spine (Phila Pa 1976). 2015:40:1660-73.

6. Kwok WY, Plevier JW, Rosendaal FR, et al. Risk factors for progression in hand osteoarthritis: a systematic review. Arthritis Care Res (Hoboken). 2013;65:552-62.

7. Zufferey P, Rebell C, Benaim C, et al. Ultrasound can be useful to predict an evolution towards rheumatoid arthritis in patients with inflammatory polyarthralgia without anticitrullinated antibodies. Joint Bone Spine. 2017:84 299-303. 
8. van der Ven $M$, van der Veer-Meerkerk M, Ten Cate DF, et al. Absence of ultrasound inflammation in patients presenting with arthralgia rules out the development of arthritis. Arthritis Res Ther. 2017;19:202.

9. Pratt $A G$, Lorenzi $A R$, Wilson $G$, et al. Predicting persistent inflammatory arthritis amongst early arthritis clinic patients in the UK: is musculoskeletal ultrasound required? Arthritis Res Ther. 2013;15:R118.

10. Nam $J$, Hensor EM, Hunt $L$, et al. Ultrasound findings predict progression to inflammatory arthritis in anti-CCP antibody-positive patients without clinical synovitis. Ann Rheum Dis. 2016;75:2060-7.

11. Rakieh C, Nam JL, Hunt L, et al. Predicting the development of clinical arthritis in anti-CCP positive individuals with non-specific musculoskeletal symptoms: a prospective observational cohort study. Ann Rheum Dis. 2015; 74:1659-66.

12. van de Stadt $L A$, Bos $W H$, Meursinge Reynders $M$, et al. The value of ultrasonography in predicting arthritis in auto-antibody positive arthralgia patients: a prospective cohort study. Arthritis Res Ther. 2010;12:R98.

13. Szkudlarek M, Court-Payen M, Jacobsen S, et al. Interobserver agreement in ultrasonography of the finger and toe joints in rheumatoid arthritis. Arthritis Rheum. 2003;48:955-62.

14. Szkudlarek M, Klarlund M, Narvestad E, et al. Ultrasonography of the metacarpophalangeal and proximal interphalangeal joints in rheumatoid arthritis: a comparison with magnetic resonance imaging, conventional radiography and clinical examination. Arthritis Res Ther. 2006;8:R52.

15. Wakefield RJ, Balint PV, Szkudlarek M, et al. Musculoskeletal ultrasound including definitions for ultrasonographic pathology. J Rheumatol. 2005;32:2485-7.

16. Torp-Pedersen ST, Terslev L. Settings and artefacts relevant in colour/power Doppler ultrasound in rheumatology. Ann Rheum Dis. 2008;67:143-9.

17. Mandl P, Naredo E, Wakefield RJ, et al. A systematic literature review analysis of ultrasound joint count and scoring systems to assess synovitis in rheumatoid arthritis according to the OMERACT filter. J Rheumatol. 2011;38:2055-62.

18. Zufferey $P$, Moller B, Brulhart $L$, et al. Persistence of ultrasound synovitis in patients with rheumatoid arthritis fulfilling the DAS28 and/or the new ACR/ EULAR RA remission definitions: results of an observational cohort study. Joint Bone Spine. 2014;81:426-32.

19. Naredo E, Collado P, Cruz A, et al. Longitudinal power Doppler ultrasonographic assessment of joint inflammatory activity in early rheumatoid arthritis: predictive value in disease activity and radiologic progression. Arthritis Rheum. 2007;57:116-24.

20. Scheel AK, Hermann KG, Kahler E, et al. A novel ultrasonographic synovitis scoring system suitable for analyzing finger joint inflammation in rheumatoid arthritis. Arthritis Rheum. 2005;52:733-43.

21. Aletaha D, Neogi T, Silman AJ, et al. 2010 rheumatoid arthritis classification criteria: an American College of Rheumatology/European League Against Rheumatism collaborative initiative. Ann Rheum Dis. 2010;69:1580-8.

22. D'Agostino MA, Wakefield RJ, Filippucci E, et al. Intra- and inter-observer reliability of ultrasonography for detecting and scoring synovitis in rheumatoid arthritis: a report of a EULAR ECSISIT task force [abstract]. Ann Rheum Dis. 2005;64(Supplement III):62.

23. McGee S. Simplifying likelihood ratios. J Gen Intern Med. 2002;17:647-50.

24. D'Agostino MA, Terslev L, Aegerter $P$, et al. Scoring ultrasound synovitis in rheumatoid arthritis: a EULAR-OMERACT ultrasound taskforce-Part 1: definition and development of a standardised, consensus-based scoring system. RMD Open. 2017;3:e000428.

25. Terslev L, Naredo E, Aegerter P, et al. Scoring ultrasound synovitis in rheumatoid arthritis: a EULAR-OMERACT ultrasound taskforce-Part 2: reliability and application to multiple joints of a standardised consensusbased scoring system. RMD Open. 2017:3:e000427.

26. Torp-Pedersen S, Christensen R, Szkudlarek M, et al. Power and color Doppler ultrasound settings for inflammatory flow: impact on scoring of disease activity in patients with rheumatoid arthritis. Arthritis Rheumatol. 2015;67:386-95.

27. van Steenbergen HW, Aletaha D, Beaart-van de Voorde LJ, et al. EULAR definition of arthralgia suspicious for progression to rheumatoid arthritis. Ann Rheum Dis. 2017:76:491-6.

28. Boer AC, Burgers LE, Mangnus $L$, et al. Using a reference when defining an abnormal MRI reduces false-positive MRI results-a longitudinal study in two cohorts at risk for rheumatoid arthritis. Rheumatology (Oxford). 2017;56: 1700-6.

29. Padovano I, Costantino F, Breban M, et al. Prevalence of ultrasound synovial inflammatory findings in healthy subjects. Ann Rheum Dis. 2016;75:1819-23.
30. Ellegaard K, Torp-Pedersen S, Holm CC, et al. Ultrasound in finger joints: findings in normal subjects and pitfalls in the diagnosis of synovial disease. Ultraschall Med. 2007;28:401-8

31. Millot F, Clavel G, Etchepare F, et al. Musculoskeletal ultrasonography in healthy subjects and ultrasound criteria for early arthritis (the ESPOIR cohort). J Rheumatol. 2011:38:613-20.

32. Kitchen J, Kane D. Greyscale and power Doppler ultrasonographic evaluation of normal synovial joints: correlation with pro- and antiinflammatory cytokines and angiogenic factors. Rheumatology (Oxford). 2015;54:458-62.

33. Fodor D, Felea I, Popescu D, et al. Ultrasonography of the metacarpophalangeal joints in healthy subjects using an $18 \mathrm{MHz}$ transducer Med Ultrason. 2015;17:185-91.

34. Machado FS, Furtado RN, Takahashi RD, et al. Sonographic cutoff values for detection of abnormalities in small, medium and large joints: a comparative study between patients with rheumatoid arthritis and healthy volunteers. Ultrasound Med Biol. 2015:41:989-98.

35. Machado FS, Natour J, Takahashi RD, et al. Sonographic assessment of healthy peripheral joints: evaluation according to demographic parameters. J Ultrasound Med. 2014:33:2087-98.

36. Hiraga M, Ikeda K, Shigeta K, et al. Sonographic measurements of lowechoic synovial area in the dorsal aspect of metatarsophalangeal joints in healthy subjects. Mod Rheumatol. 2015;25:386-92.

37. van Steenbergen HW, Mangnus L, Reijnierse M, et al. Clinical factors, anticitrullinated peptide antibodies and MRI-detected subclinical inflammation in relation to progression from clinically suspect arthralgia to arthritis. Ann Rheum Dis. 2016;75:1824-30.

\section{Ready to submit your research? Choose BMC and benefit from:}

- fast, convenient online submission

- thorough peer review by experienced researchers in your field

- rapid publication on acceptance

- support for research data, including large and complex data types

- gold Open Access which fosters wider collaboration and increased citations

- maximum visibility for your research: over $100 \mathrm{M}$ website views per year

At $\mathrm{BMC}$, research is always in progress.

Learn more biomedcentral.com/submissions 